\title{
Printed 2D Proton Sensor for In-Situ Measurement in Glue Lines ${ }^{+}$
}

\author{
Matic Krivec ${ }^{1}$, Thomas Stockinger ${ }^{2}$, Johanna Zikulnig ${ }^{1, *}$, Friedrich Eibensteiner ${ }^{3}$ and \\ Uwe Müller 4 \\ 1 CTR Carinthian Tech Research AG, Europastraße 12, 9524 Villach, Austria; krivec.matic@gmail.com \\ 2 Soft Matter Physics, Johannes Kepler University, Altenbergerstraße 69, 4040 Linz, Austria; \\ Thomas.Stockinger@jku.at \\ 3 Prelonic Technologies, Lannergasse 7, 4020 Linz, Austria; f.eibensteiner@prelonic.com \\ 4 Kompetenzzentrum Holz GmbH, Altenbergerstraße 69, 4040 Linz, Austria; u.mueller@wood-kplus.at \\ * Correspondence: johanna.zikulnig@ctr.at; Tel.: +43-(0)-4242-56300-276 \\ + Presented at the Eurosensors 2018 Conference, Graz, Austria, 9-12 September 2018. \\ Published: 4 December 2018
}

\begin{abstract}
A screen printed $\mathrm{pH}$ sensor was developed using a PANI layer as a proton sensitive material for the in-situ measurements of matrix cross-linking. The sensor showed a linear response in a broad $\mathrm{pH}$ range (3-10) and had an evident cross-talk to $\mathrm{Cl}^{-}$ions. Preliminary in-situ measurements showed a substantial signal change during the cross-linking process.
\end{abstract}

Keywords: printed $\mathrm{pH}$ sensor; PANI layer; in-situ proton sensing

\section{Introduction}

Wood industry uses conventional methods (i.e., pulling, bending, and ripping) for the characterization of their products. These methods are normally cost-, material- and time-consuming, ex-situ, destructive and incompatible with the state-of-the-art connectivity and data evaluation procedures that enable the modern industrial Internet of Things (IoT). The performance of wood composites, the mechanical core of many wood-related products, is strongly influenced by the type and degree of cross-linking of the matrix. The in-situ measurement of temperature, protonconcentration and impedance in the press-moulding process can greatly improve the quality of the products. These sensors have to be 2D (easy integration), robust (withstanding the pressures), cheap and have to act as a part of the matrix material without any mechanical or any other influence on the end-product (the sensors will not be removed after processing). The traditional sensor technologies (e.g., silicon-based) do not fulfill most of the requirements stated above; therefore, alternative sensors such as printed ones not only complement the silicon technology but also open new possibilities and applications that were so far not feasible [1].

Printed electronics (PE) enables the development of sensors and smart systems with unique characteristics such as flexibility, conformability, transparency, biocompatibility and the possibility of easy integration and even fabrication of sensing parts over a large area. Moreover, the use of additive processes allows PE devices to be fabricated waste-free and at very low-cost [1]. Printed sensors were identified to be the most suitable sensor-platform for the undistorted, real-time and insitu measurements of cross-linking kinetics, curing and aging of glue joints, coating and composites. Although some studies already show the high potential of printed sensors for the in-line impedance detection [2], printed $\mathrm{pH}$ sensors selectively detecting proton formation can give an in-depth information of the chemical cross-linking. 
In the present paper, a screen printed proton sensor based on polyaniline (PANI) has been developed for the in-situ measurement of the cross-linking of glue lines inside the wood composite. The sensors were characterized with standard buffer solutions and first preliminary in-situ measurements were conducted.

\section{Materials and Methods}

\subsection{Sensor Development}

A three electrode structure was manufactured via screen printing of Ag (DuPont 5028, Du Pont Limited, Michigan, USA), carbon (SD 2843 HAL, Lackwerke Peters GmbH + Co KG, Kempen, Germany) and insulator (Thermo-Jet ${ }^{\circledR}$, Proell KG, Weißenburg in Bayern, Germany), as seen in Figure 1a. A proton-sensitive polyaniline (PANI) layer was deposited on the working electrode with the selective electropolymerization, according to the literature [3]. Briefly, printed electrodes were positioned into the 3D-printed holder, as shown in Figure 1b. $0.1 \mathrm{M}$ monomeric aniline prepared in $1 \mathrm{M} \mathrm{HCl}$ was deposited over the electrodes and a PANI electropolymerization was performed by cyclic voltammetry (CV) from $-0.2 \mathrm{~V}$ to $1.0 \mathrm{~V}$ using portable potentiostat (HSTAT 4000P, DropSens, Llanera, Spain). After first $12 \mathrm{CV}$ cycles, the depleted solution was removed and a fresh aniline solution was deposited again, followed by another $12 \mathrm{CV}$ cycles. The growth of the oxidation peak at the potential of $0.35 \mathrm{~V}$ indicates the formation of the PANI layer.

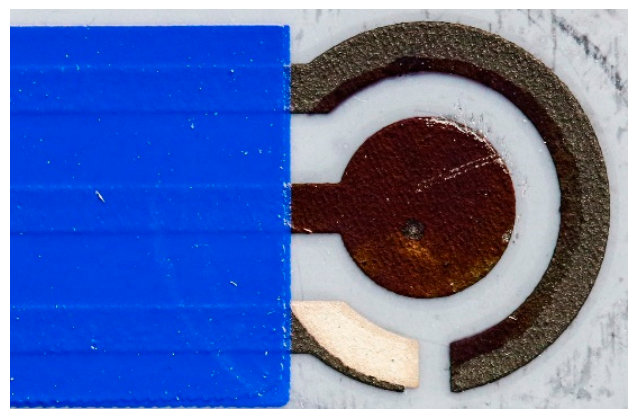

(a)

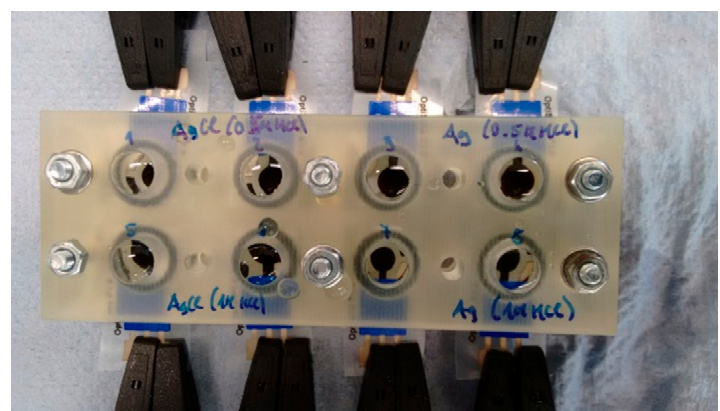

(b)

Figure 1. (a) A three-electrode sensor structure manufactured with screen printing of silver (contact pads, connections and reference electrode) and carbon (working and auxiliary electrode); (b) set-up of the parallel $\mathrm{pH}$ calibration measurements in a 3D printed measurement housing.

\subsection{Sensor Characterization}

The performance of printed sensors was characterized with the parallel $\mathrm{pH}$ measurements (Figure 1b) of open circuit potential (OCP) between the working and the reference electrode. Firstly, measurements with standard $\mathrm{pH}$ solutions between $\mathrm{pH} 2$ and 10 were performed. The buffers had different elemental structure: $\mathrm{pH} 2-$ citric acid, $\mathrm{HCl}, \mathrm{NaCl} ; \mathrm{pH} 3$ and $4-$ citric acid, $\mathrm{NaOH}, \mathrm{NaCl} ; \mathrm{pH}$ 5 and $6-$ citric acid, $\mathrm{NaOH} ; \mathrm{pH} 7$ and $8-\mathrm{KH}_{2} \mathrm{PO}_{4}, \mathrm{NaOH}$; $\mathrm{pH} 9$ and $10-$ boric acid, $\mathrm{NaOH}, \mathrm{KCl}$. Due to the potential cross-sensitivity of other ions, such as $\mathrm{Cl}^{-}$, on the PANI layer [4], a second reference buffer solution (Mcllvains solution) with fixed elemental structure $\left(\mathrm{Na}_{2} \mathrm{HPO}_{4}\right.$, citric acid) was prepared and used for the calibration measurements.

\subsection{In-Situ Measurements}

The in-situ measurements were performed according to the scheme in Figure 2. A woodensubstrate was covered with the glue (Primere 10F152, Metadynea Austria GmbH, Krems an der Donau, Austria) and the proton sensor as well as the thermocouple (type K) was positioned on the top of the glue surface. The proton sensor was turned upside down to ensure the direct contact of the glue with the PANI surface. The sensors were covered with another wood part, put into the hot-press and a 40-min cross-linking procedure was conducted with a constant pressure of 20 bars and temperature of $120^{\circ} \mathrm{C}$ (ramp: $\left.5^{\circ} \mathrm{C} / \mathrm{min}\right)$. 


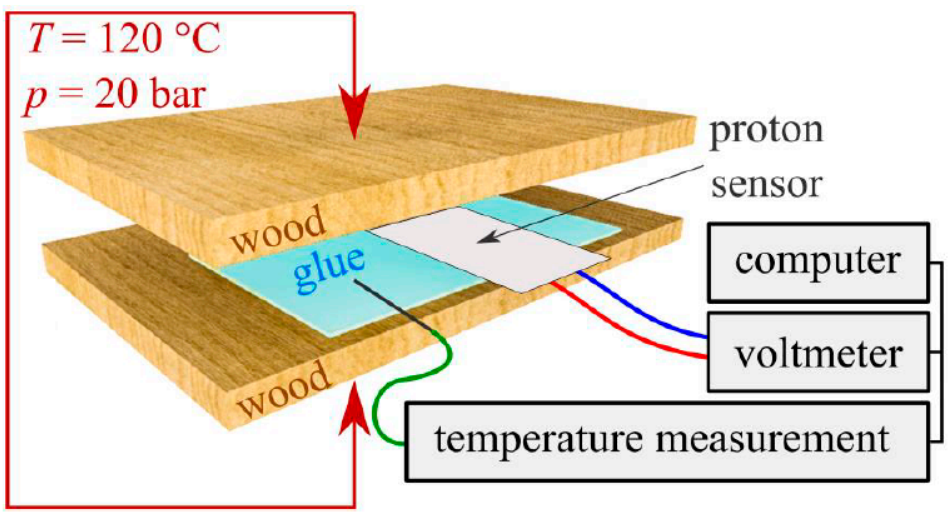

Figure 2. Schematic presentation of the experimental setup for the in-situ measurements of crosslinking.

\section{Results and Discussion}

Measurements with standard $\mathrm{pH}$ solutions (Figure 3a) show a linear response of $\mathrm{pH}$ values between 2-4 and 9-10, while the measured values are somehow shifted but retain the same slope between $\mathrm{pH} 5-8$. The latter was assigned to the effect of the $\mathrm{Cl}^{-}$ions which evidently also react with the PANI surface and increase the signal. A second round of testing using the McIlvains buffer solution free of $\mathrm{Cl}^{-}$supported our previous suggestion (Figure $3 \mathrm{~b}$ ); the sensor possessed a perfect linear response between $\mathrm{pH} 3$ and $8\left(\mathrm{R}^{2}=0.993\right)$ with high sensitivity $(70 \mathrm{mV} / \mathrm{pH})$.

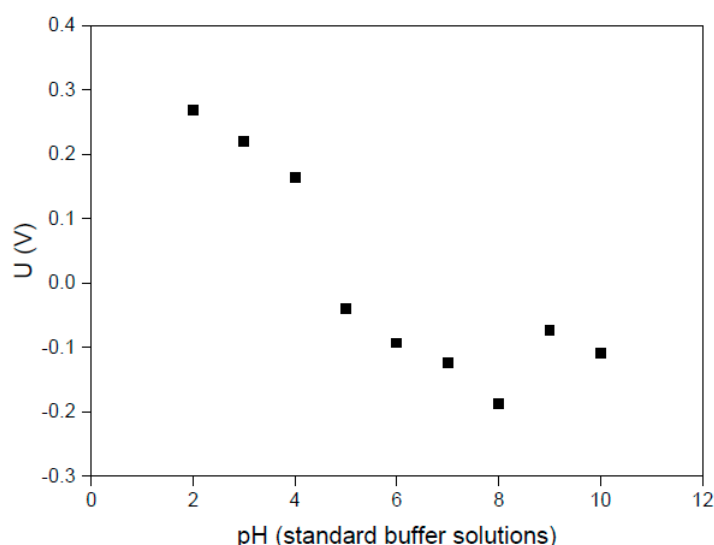

(a)

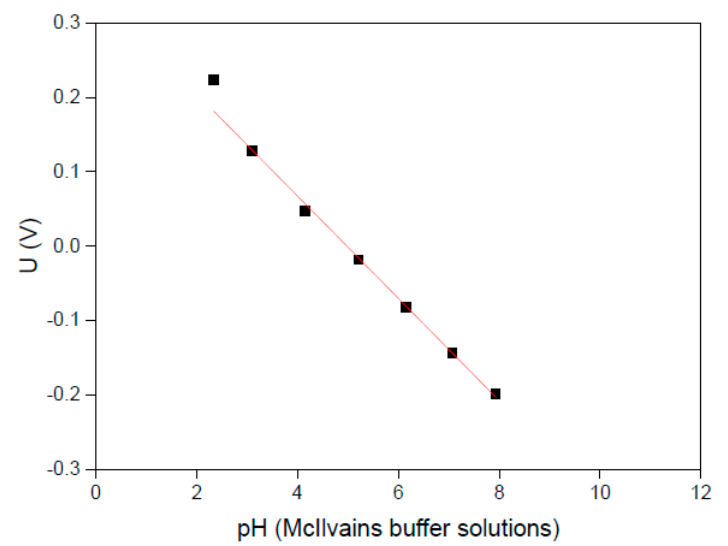

(b)

Figure 3. Characterization of the printed $\mathrm{pH}$ sensor with standard $\mathrm{pH}$ buffer solutions: (a) commercial pH buffers with different elemental structure and (b) Mcllvains buffer solutions.

For the in-situ testing, the sensor was positioned on the wooden-substrate, as shown in Figure $4 \mathrm{a}$, that was glued to another one with a cross-linking of binder with and without the hardener. The preliminary results (Figure $4 \mathrm{~b}$ ) show the evident signal of the printed sensors with the maximal absolute signal change of $140 \mathrm{mV}$. Although the signal is evidently influenced by the temperature profile, the additional peak in the case of gluing with hardener suggests the chemical formation of protons after reaching $70^{\circ} \mathrm{C}$. This observation is also in agreement with the cross-linking chemistry, as the formation of the $\mathrm{H}^{+}$is initiated above $60^{\circ} \mathrm{C}$. 


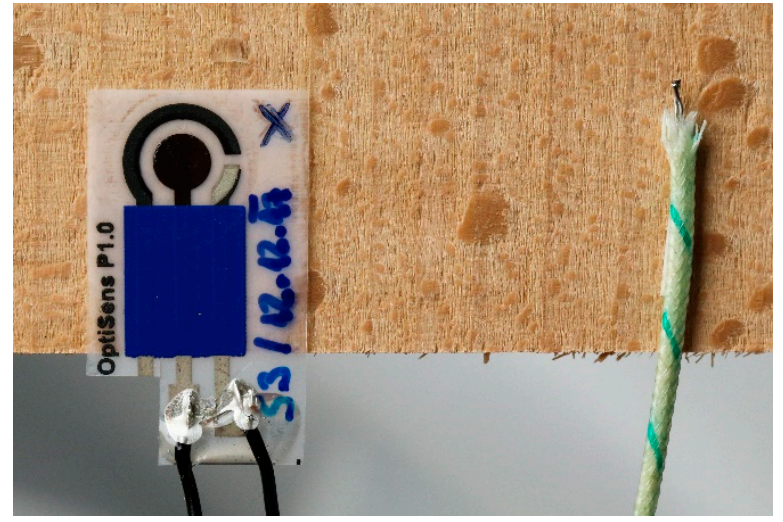

(a)

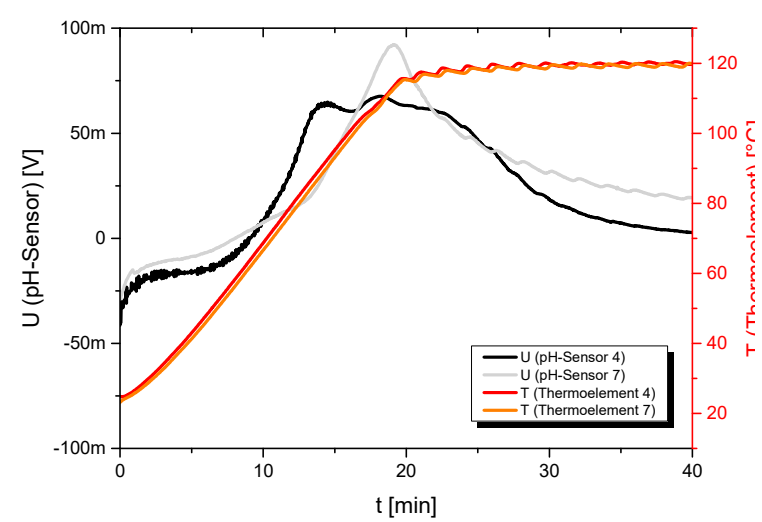

(b)

Figure 4. (a) Integration of the printed proton sensor for the in-situ measurements of cross-linking and the thermocouple; (b) in-situ measurements of $\mathrm{pH}$ and temperature during the hot-press gluing process.

\section{Conclusions}

The 2D printed proton sensor was manufactured using screen printing of the three-electrode structure and subsequent selective electropolymerization of PANI on top of the working electrode. The sensor shows a linear response between $\mathrm{pH} 3$ and 8, and has a noticeable cross-sensitivity to $\mathrm{Cl}^{-}$ ions. The in-situ measurements show a significant signal after $15 \mathrm{~min}$ hot-pressing procedure which corresponds to the formation of protons during the cross-linking of closed glue joints.

Author Contributions: M.K., J.Z. and T.S. conceived and designed the experiments; F.E. manufactured the electrode structures; M.K. fabricated the PANI layer; M.K. and J.Z. performed the pH calibration experiments; T.S. performed the hot-press measurements; M.K. and U.M. analyzed the data; M.K. and T.S. wrote the paper.

Funding: The work described in this publication was funded by the OptiSens project 855869 of the Austrian Research Promotion Agency (FFG) and the R\&D Program COMET-Competence Centers for Excellent Technologies by the Austrian Federal Ministries of Transport, Innovation and Technology (BMVIT), of Economics and Labour (BMWA) managed by the FFG.

Conflicts of Interest: The authors declare no conflict of interest.

\section{References}

1. Mattana, G.; Briand, D. Recent advances in printed sensors on foil. Mater. Today 2016, 19, 88-99, doi:10.1016/j.mattod.2015.08.001.

2. Alkin, K.; Stockinger, T.; Zirkl, B.; Stadlober, B.; Bauer-Gogonea, S.; Kaltenbrunner, M.; Bauer, S.; Müller, U.; Schwödiauer, R. Paper-based printed impendance sensors for water sorption and humidity analysis. Flex. Print. Electron. 2017, 2, doi:10.1088/2058-8585/aa5eeb.

3. Guinovart, T.; Valdes-Ramirez, G.; Windmiller, J.R.; Andrade, F.J; Wang, J. Bandage-Based Wearable Potentiometric Sensor for Monitoring Wound pH. Electroanalysis 2014, 26, 1-9, doi:10.1002/elan.201300558.

4. Li, X.; Ju, M.; Li, X. Chlorine ion sensor based on polyaniline film electrode. Sens. Actuators B Chem. 2004, 97, 144-147, doi:10.1016/j.snb.2003.08.006.

(C) 2018 by the authors. Licensee MDPI, Basel, Switzerland. This article is an open access article distributed under the terms and conditions of the Creative Commons Attribution (CC BY) license (http://creativecommons.org/licenses/by/4.0/). 